\title{
El Sistema Nacional de Regalías y su impacto en la inversión social del departamento de La Guajira
}

The national system of royalties and its impact on social investment in the department of Guajira

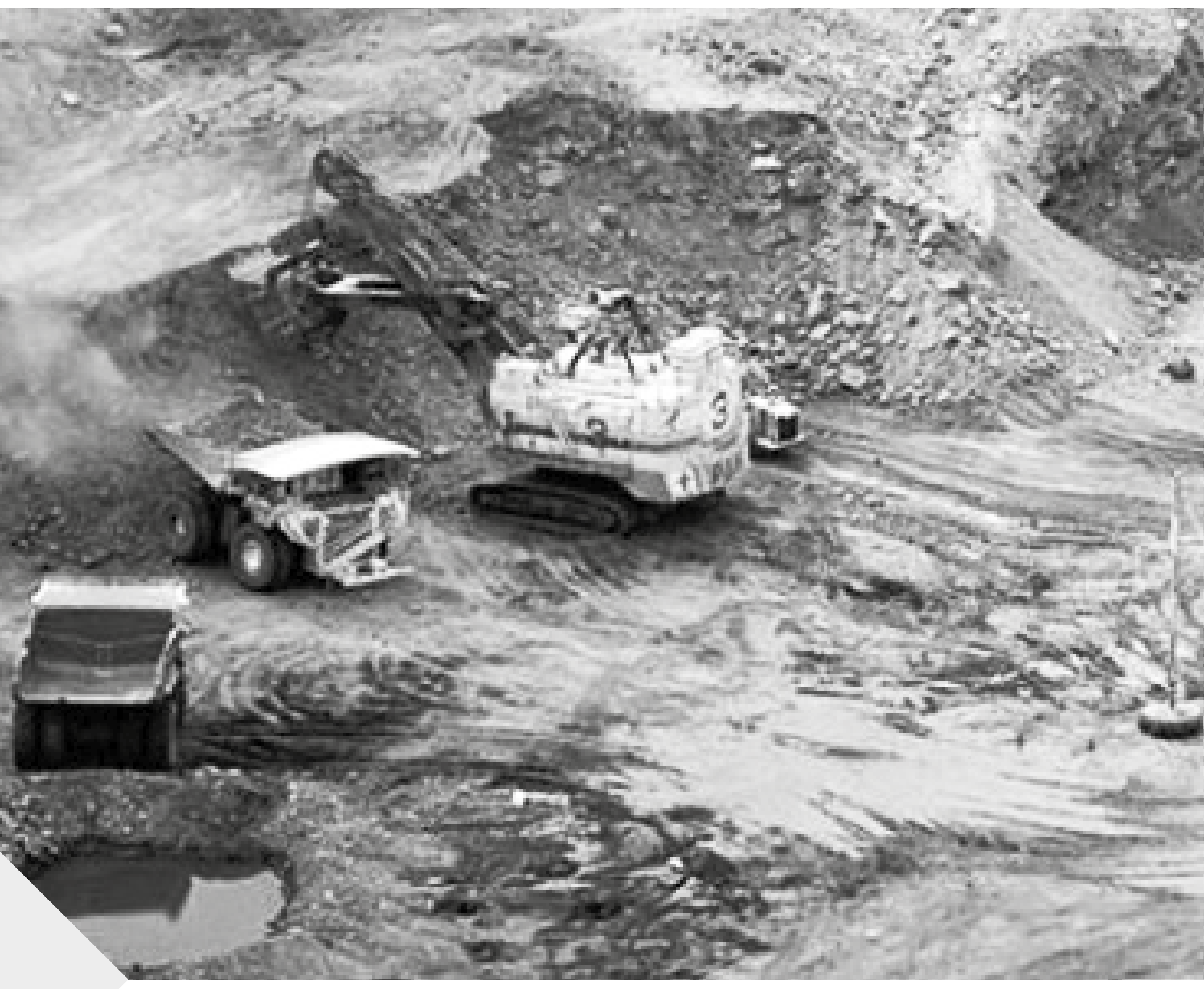




\title{
El Sistema Nacional de Regalías y su impacto en la inversión social del departamento de La Guajira'
}

\section{The national system of royalties and its impact on social investment in the department of Guajira}

\author{
Edwin Salas Solano², Darcy Luz Mendoza Fernández³, \\ Danny Daniel López Juvinao ${ }^{4}$
}

Universidad de La Guajira, Riohacha, Colombia

Artículo recibido en julio de 2016; artículo aceptado en noviembre de 2016

Citación del artículo: Salas, E. Mendoza, D. \& López, D. (2017). El sistema nacional de regalías y su impacto en la inversión social del departamento de La Guajira. I+D Revista de Investigaciones, 9 (1). pp. 82 - 92

\begin{abstract}
Resumen
El propósito del presente artículo es determinar el impacto que ha tenido el Sistema General de Regalías en la inversión social del departamento de La Guajira. Esta investigación, de tipo descriptivo, fue realizada sobre realidades de hecho. Se obtuvo información de la ciudadanía radicada en cada uno de los municipios objeto de estudio y de los representantes legales de las diferentes empresas que aportan regalías al departamento de La Guajira, y se evidenció el impacto que tienen las regalías y su repercusión en la inversión social en la población.Se concluyó que existen una serie de irregularidades, tales como faltas en la contratación del personal, manejo indebido del presupuesto local, problemas financieros de las instituciones públicas, construcción y ejecución de los planes de desarrollo municipal y departamental, problemas en la documentación legal de los registros estadísticos, incumplimiento gubernamental con las comunidades, problemas en la formulación y ejecución de proyectos sociales, entre otros.
\end{abstract}

Palabras clave: Ley de regalías, inversión social, regalías mineras, políticas públicas.

\footnotetext{
Abstract

The purpose of this article is to determine the impact of the general system of royalties on social investment in the department of La Guajira. This research is of descriptive type, realized on realities of fact. Information was obtained from the citizens residing in each of the municipalities under study and from the legal representatives of the different

1. Artículo de investigación científica y tecnológica, enfoque cuantitativo, resultado del proyecto de investigación El sistema nacional de regalías y su impacto en la inversión social del departamento de La Guajira (culminado), perteneciente al área de Ciencias económicas y administrativas, subárea de Tributación y Política Fiscal, desarrollado en el grupo de investigación E'itaja y financiado por la Universidad de La Guajira de la ciudad de Riohacha (Colombia). Dirección: Km 5 Vía a Maicao, Riohacha. PBX: (5) 7282729. Fecha de inicio: enero 2013, fecha de terminación: octubre 2015.

2. Licenciado en Contaduría Pública (Universidad Cooperativa de Colombia, seccional Bucaramanga). Magister en Tributación y Política Fiscal (Universidad de Medellín). Docente Universidad de La Guajira, Colombia. Grupo de investigación: E'itaja. Dirección: Calle 32 \# 7B - 66, Riohacha, La Guajira. Correo institucional: esalas@uniguajira.edu.co

3. Licenciada en Administración de Empresas (Universidad de La Guajira). Doctora en Ciencias Gerenciales (Universidad Rafael Belloso Chacín. Venezuela). Profesora de planta. Universidad de La Guajira. Riohacha, Colombia. Grupo de investigación: E'itaja. Dirección: Calle 32 \# 7B - 66, Riohacha, La Guajira. Correo institucional: dmendoza@uniguajira.edu.co

4. Ingeniero en Minas (Universidad Pedagógica y Tecnológica de Colombia). Doctor en Ciencias Gerenciales (Universidad Rafael Belloso Chacín. Venezuela). Profesor de planta. Universidad de La Guajira. Riohacha, Colombia. Grupo de investigación: Contacto con la comunidad. Dirección: Carrera 5 \# 21 - 61, Maicao, La Guajira. Celular: 3013711602. Correo institucional: dlopezj@uniguajira.edu.co
} 
companies that contribute royalties to the department of La Guajira, and showed the impact of royalties and their impact on social investment In the population. It was concluded that there are a number of irregularities, such as lack of staff recruitment, mismanagement of the local budget, financial problems of public institutions, construction and execution of municipal and departmental development plans, problems in the legal documentation of Statistical records, government noncompliance with communities, problems in the formulation and execution of social projects, among others.

Key words: Royalties law, social investment, mining royalties, public policy.

Las regalías son una fuente importante de financiación para el desarrollo territorial, que deben administrarse siguiendo los principios de transparencia, eficiencia, impacto, equidad y sostenibilidad. También se consideran una contraprestación económica que recibe el Estado por la explotación de un recurso natural no renovable, cuya producción se extingue en el transcurso del tiempo. Las regalías son un beneficio económico importante para el Estado y sus entidades territoriales.

El departamento de La Guajira presenta un rezago histórico en economía, tecnología y en general en todos los aspectos del desarrollo humano. También tiene unos indicadores de salud y educación que están entre los peores del país y por ello es necesario revisarlos antes de evaluar sus actuales condiciones económicas y su participación en el contexto regional, nacional y mundial. En La Guajira, el nivel de participación del producto interno bruto (PIB) a valores constantes, en promedio es de un $0,5798 \%$, levemente superior a la participación corriente. Esta participación se ha presentado durante los últimos seis años. La realidad que se vive en el departamento es delicada, ninguno de los municipios del departamento consume agua potable, y a nivel regional el departamento es el último en coberturas nominales de agua potable y alcantarillado, con un $72 \%$ y $46 \%$ respectivamente.

La medición de la pobreza por medio de la metodología de las necesidades básicas insatisfechas, NBI, es el método más extendido en América Latina; el NBI define a los pobres como aquellas personas que tienen carencias, privaciones 0 necesidades básicas insatisfechas, sobre todo en materia de servicios públicos: vivienda, saneamiento básico y educación. Según el Departamento Administrativo Nacional de Estadística, DANE, la metodología de NBI busca determinar, con ayuda de algunos indicadores simples, si las necesidades básicas de la población se encuentran cubiertas. Los grupos que no alcancen un umbral mínimo fijado, son clasificados como pobres.

Los indicadores simples seleccionados son viviendas inadecuadas, hogares con hacinamiento crítico, viviendas con servicios inadecuados, hogares con alta dependencia económica y hogares con niños en edad escolar (7-12 años) que no asisten a la escuela.

En Colombia, los grandes esfuerzos sin precedentes de los gobiernos del decenio anterior para mejorar la calidad de vida de los ciudadanos, no han sido suficientes para lograr las metas, ya que en la actualidad una persona de cada tres, en todo el país, carece de agua potable en cantidad y calidad, imprescindibles para la salud y una mejor condición humana. Adicionalmente a lo anterior, se reconoce que los residuos líquidos son causa del deterioro de las fuentes hídricas, y que el uso de tecnologías apropiadas y la construcción de estaciones de depuración de aguas residuales podrían ser importantes factores de progreso en Colombia.

Con esta investigación se trata de establecer si las políticas públicas relacionadas con el sistema general de regalías han sido coherentes con los objetivos establecidos que subyacen en estos recursos financieros, como es que sean bien usados por las regiones y municipios productores de recursos naturales no renovables. Además se busca determinar si las regalías del carbón en el departamento de La Guajira están siendo invertidas de manera directamente proporcional a la inversión social y si estas contribuyen al bienestar social de la comunidad, teniendo en cuenta que cambiaron las reglas de juego del Sistema General de Regalías, razón por la cual, después de recibir de manera directa las regalías de carbón y gas La Guajira se enfrenta a la nueva institucionalidad que genera la aprobación del acto legislativo n. ${ }^{\circ} 05$ de 2001 y el decreto 4923 de 2011.

\section{Las regalías y un poco de su historia}

La historia reciente de las regalías en Colombia se inicia con la Constitución Política de Colombia (1991) artículo 360 , que dispone una "contraprestación económica a título de regalía" por la explotación de un recurso natural no renovable, y el derecho de los departamentos o municipios en donde se encuentran dichas explotaciones, así como de los puertos por donde se transporten esos recursos o los productos derivados de estos, a participar en tales regalías. 
Para los recursos provenientes de las regalías no asignados a los departamentos y municipios involucrados directamente, el artículo 361 propuso la creación de un fondo nacional a fin de destinarlos a los demás entes territoriales con fines de promoción de la minería, preservación del medio ambiente y financiación de proyectos regionales de inversión. En aplicación de lo anterior, la Ley 141 de 1994 (Ley de Regalías) creó el Fondo Nacional de Regalías (FNR) y reguló el derecho del Estado a percibir regalías, su liquidación y distribución.

Por su parte, diferentes estudios que se han realizado para determinar la situación del país han encontrado que el aumento de ingresos de las regalías no se ha visto reflejado necesariamente en un mayor desarrollo económico, y que se han generado fallas en la administración de dichos recursos, además de corrupción. En un estudio sobre el impacto de las regalías en la inversión de los entes territoriales en Colombia, Benavides et al. (2000) encontraron que los grandes recursos que se han generado por regalías llevan a una situación fiscal insostenible, dado que los ingresos por este concepto incrementan la dependencia fiscal y limitan la autonomía política de los alcaldes. Además, encuentran que el gasto que se realiza con los dineros provenientes de las regalías no se destina a proyectos prioritarios, sino que estos dineros se utilizan en proyectos que ni siquiera tuvieron estudio de impacto previo, quitándole así a sectores como la salud la importancia que se merecen.

Por otro lado, Gaviria, Zapata y González (2002), en un estudio sobre regalías y finanzas públicas en el Casanare, concluyen que los territorios petroleros empeoraron su situación fiscal al recibir regalías, ya que a medida que incrementan sus recursos, los gastos y el endeudamiento aumentan, generando una alta dependencia de las regalías, aspecto a tener en cuenta para que los departamentos no dependan de estas.

\section{Destinación de las regalías}

Los recursos que reciben las entidades territoriales por concepto de regalías tienen como propósito cubrir las necesidades básicas de la población. Debido a esto, las asignaciones se orientan principalmente a la inversión en proyectos que permitan que más colombianos tengan acceso a los servicios de educación básica, salud, agua potable y alcantarillado.

La Ley de Regalías establece la destinación específica, que tanto los departamentos y municipios productores y portuarios deben darle a su participación de las regalías por la explotación de recursos naturales no renovables.
Por otra parte, en el Decreto 1747 de 1995 se establecen los niveles mínimos de cubrimiento de los servicios básicos que deben ser alcanzados por los departamentos y municipios con la participación de las regalías; estas coberturas mínimas son:

\section{Tabla 1}

\section{Coberturas mínimas de servicios básicos}

\begin{tabular}{cc}
\hline Coberturas mínimas & Porcentaje \\
\hline Mortalidad infantil & $1 \%{ }^{4}$ \\
Salud de la población & $100 \%$ \\
Educación básica & $90 \%$ \\
Agua potable & $70 \%$ \\
Alcantarillado & $70 \%$ \\
\hline
\end{tabular}

\section{Fuente: Decreto 1747 de 1995}

A pesar de las todas referencias nombradas anteriormente, se podría decir que en contraste con lo previamente descrito, se presentan casos de países que han logrado manejar positivamente el uso de las regalías y enfocar su destinación a inversiones prioritarias con buena planeación. Este es el caso de Noruega, que logró generar un crecimiento económico sostenido por un buen manejo de los recursos generados por la producción de petróleo y logró constituir un fondo financiero con las utilidades, impuestos y regalías.

Volviendo al contexto local, Meisel (2007) expone el caso de las regalías para el departamento de La Guajira, haciendo un análisis de las regalías de carbón y gas; el resultado muestra que el atraso económico de La Guajira antes del gas y el carbón era tan grande, que el rápido desarrollo de un solo sector no es suficiente para dinamizar toda la economía y así producir un buen nivel de bienestar para sus habitantes.

\section{El departamento de La Guajira y las regalías}

Según Serrano, Martínez y Acened (2016), la minería en Colombia está considerada como una de las actividades económicas con mayor crecimiento y desarrollo en los últimos años; concretamente, el sector carbonífero ha tenido un notable impulso desde los años ochenta, principalmente en los departamentos del Cesar y La Guajira, lo que ha originado ingresos significativos para el progreso del país.

El departamento de La Guajira tiene una extensión de 20 $848 \mathrm{~km}^{2}$, que representan el 15,25\% de la región Caribe colombiana y el $1,76 \%$ de la superficie del país. Está conformado por 15 municipios, 57 corregimientos, 49 inspecciones y 10 caseríos. 
Este departamento presenta un rezago histórico en economía, tecnología y en general en todos los aspectos del desarrollo humano. Sus indicadores de salud y educación son de los más bajos del país y por ello es necesario revisarlos antes de evaluar las actuales condiciones económicas, y su participación en el contexto regional, nacional y mundial. El nivel de participación del PIB a valores constantes en los últimos años es, en promedio de $0,5798 \%$, levemente superior a la participación corriente.

En La Guajira, los niveles de pobreza son superiores al promedio regional y nacional. En 2013 presentó índices de NBI del 64,5\%, mientras la región Caribe presenta el $52,4 \%$ y la nación el $37 \%$. Con relación a los índices de miseria, el departamento presenta $41,8 \%$, la región $28 \%$ y el país $16 \%$. Se puede decir que un habitante promedio de La Guajira recibe un $45 \%$ menos de ingreso per cápita que el resto de los colombianos; estas variaciones en los porcentajes mencionados son irregulares, indicando que el crecimiento global de la economía departamental también ha sido y es menor que el de la nacional en periodos similares, dejando en evidencia que la estructura económica del departamento es básicamente pobre, débil y con mínima participación en el contexto nacional.

La base económica de La Guajira está constituida por la minería, más que todo carbón y la sal, y el sector agropecuario. En menor medida, pero con un buen potencial, están el turismo y la pesca. Las exportaciones de carbón de La Guajira se expandieron rápidamente. Sin embargo, pese a que el departamento recibe millonarias regalías por la explotación del carbón, un alto porcentaje de la población tiene sus necesidades básicas insatisfechas, tal como se develó anteriormente.

Esto indica que, los millones de dólares que ha recibido el departamento en las últimas dos décadas se han desviado por la corrupción y la ineficiencia, o ambas cosas, y por esa razón La Guajira tiene bajos indicadores sociales (Meisel, 2007).

De este modo, el departamento de La Guajira, con el cambio surgido de la nueva Ley de Regalías (Ley 530 de 2012) por la cual se regula la organización y el funcionamiento del Sistema General de Regalías, tendría inconvenientes, puesto que si a lo largo de la existencia de dichas regalías La Guajira ha recibido millones de dólares y las condiciones sociales no han sido las mejores, ahora lo que se espera es que la situación social de sus habitantes empeore, porque se recibirán menos recursos financieros.
Ahora bien, considerando que por causa de la corrupción los recursos que se han recibido van a parar a las arcas individuales de los dirigentes del departamento, también se podría esperar que lo poco a recibir sea bien invertido; de esta forma, la Ley General de Regalías tiende a acentuar aún más las disparidades entre las regiones desarrolladas y pujantes del país, frente a las más rezagadas y atrasadas.

\section{Inversión social y regalías en La Guajira, una realidad incierta}

Desde la concepción de las regalías en el departamento, siempre se ha dicho que estas son invertidas en las necesidades básicas insatisfechas, tal como lo requiere la normatividad vigente para tal fin.

La Tabla 2 muestra el total de regalías recibidas por el departamento de La Guajira en el periodo 2004-2013; según estos datos, el departamento recibió más de dos mil millones de dólares, una cifra bastante significativa para una región con tantas necesidades básicas insatisfechas.

\section{Tabla 2}

Giros de regalías del carbón en el departamento de La Guajira

\begin{tabular}{cc}
\hline Año & Valores $\mathbf{( \$ )}$ \\
\hline $\mathbf{2 0 0 4}$ & 70651127165 \\
$\mathbf{2 0 0 5}$ & 148441137620 \\
$\mathbf{2 0 0 6}$ & 218641571133 \\
$\mathbf{2 0 0 7}$ & 198274811729 \\
$\mathbf{2 0 0 8}$ & 356350288136 \\
$\mathbf{2 0 0 9}$ & 483364384868 \\
$\mathbf{2 0 1 0}$ & 242801196274 \\
$\mathbf{2 0 1 1}$ & 396121066844 \\
$\mathbf{2 0 1 2}$ & 123727703473 \\
$\mathbf{2 0 1 3}$ & 197381440541 \\
Total & 2434754726783 \\
\hline
\end{tabular}

Fuente: Sistema de Información Minero Colombiano, Simco (2015)

Por otra parte, el departamento presenta un escaso desarrollo social con altos índices de necesidades básicas insatisfechas, preocupantes cifras que aseveran las condiciones de pobreza, hacinamiento, deserción escolar, desempleo, analfabetismo, y bajas coberturas de educación básica secundaria y media, factores determinantes que lo clasifican como de alto riesgo, lo cual requiere de estrategias de intervención eficaces y sostenibles, además de inversiones suficientes para generar un desarrollo local. 
Eneste sentido, según datos de planeación departamental las regalías han tenido la siguiente inversión:

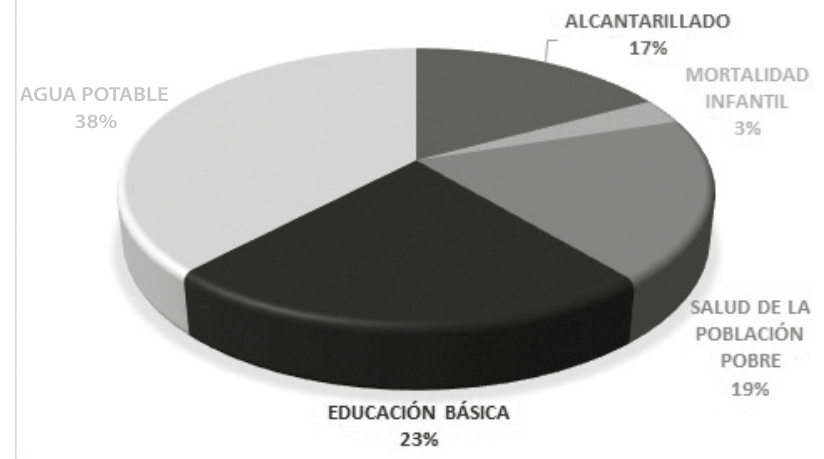

Figura 1.

Inversión de regalías en Necesidades Básicas 2007-2013. Fuente: Plan de desarrollo departamental 2012 - 2015 "La Guajira Primero".

Como se puede notar en la Figura 1, el $38 \%$ de las regalías han sido invertidas en agua potable, sin embargo, La Guajira todavía no cuenta con agua potable; y si no se presenta una profunda inversión social y si disminuyen las regalías, la situación será peor.

En este mismo orden de ideas, el $23 \%$ de los recursos están invertidos en educación básica. Según el DANE, en Colombia para los primeros años de la década las coberturas en educación básica se encontraban alrededor del $84 \%$ y desde el año 2005 a nivel nacional se hizo un esfuerzo por aumentar esta cobertura al 100\% para universalizar la educación primaria, sin embargo, la tasa de analfabetismo más alta de todo el país fue para el departamento de La Guajira.

Así las cosas, después de recibir de manera directa las regalías de carbón y gas, ahora La Guajira se enfrenta a la nueva institucionalidad que genera la aprobación del Acto legislativo n. 05 de 2011 y el Decreto 4923 de 2011. Estas nuevas reglas de juego están cimentadas en que las regalías no fueron bien usadas por las regiones y los municipios productores de recursos naturales no renovables.

Ahora, todas las regiones participan, no solo las productoras, y se crea un mecanismo de ahorro para la sostenibilidad futura, con el cual se pretende: financiar proyectos de impacto regional-competitividad; contribuir a la convergencia regional y a aliviar la pobreza; fortalecer los recursos dedicados a ciencia, tecnología e innovación; y administrar los recursos bajo un sistema de organismos colegiados, abandonando el sistema de asignación de recursos a partir de la discrecionalidad del gobernante (contribuir al buen gobierno).

\section{Metodología}

En consonancia con los aspectos estructurales de este estudio y a sus objetivos, la investigación es de tipo descriptivo, puesto que se trabaja sobre realidades de hecho, y se registra, analiza e interpreta la situación problema que se viene presentando en el municipio de Riohacha con la inversión de las regalías derivadas de la explotación de los recursos naturales no renovables.

Es importante destacar que para efecto del análisis que demuestre la incidencia social de los recursos de regalías en la satisfacción de las necesidades básicas de los habitantes del municipio de Riohacha, se tendrá en cuenta la metodología de medición de la pobreza denominada NBI.

La investigación es de tipo no experimental, transeccional, con diseño de campo. Según Hernández, Fernández y Baptista (2005), la investigación de tipo no experimental se realiza sin manipular deliberadamente la variable, y los fenómenos se observan tal como se dan en su contexto natural, para después analizarlos.

El estudio, además, se inscribe en la modalidad de campo, debido a que está basado en informaciones obtenidas directamente de la realidad, de tal manera que se comprobó la inversión social con recursos de las regalías recibidas por el departamento. También, la investigación se en un entorno real, donde la comunidad del departamento de la Guajira constituye el universo de la investigación.

En la presente investigación se trabajó con dos poblaciones, la primera corresponde a los habitantes de La Guajira (ver Tabla 3), los cuales constituyen las unidades informantes: $y$, la segunda población objeto de estudio está constituida por los dirigentes del departamento (ver Tabla 4).

En el primer caso, el tamaño de la muestra obtenido fue de 118 habitantes, y para la segunda población se tuvieron en cuenta 16 directivos del ente departamental y municipal.

Se diseñaron dos cuestionarios: el primero, dirigido a directivos de La Guajira, fue diseñado en función de veintiocho ítems, con respuestas tipo Likert; y el segundo, dirigido a la comunidad del departamento, está constituido por veinte ítems con respuestas dicotómicas sobre los beneficios sociales derivados de las regalías.

Utilizando el procedimiento a través del programa estadístico prediseñado para tal fin, el cual está 
Tabla 3

Población de los municipios de La Guajira

\begin{tabular}{ccc}
\hline Ítem & Municipios & Población 2013 \\
\hline $\mathbf{1}$ & Riohacha & 236969 \\
$\mathbf{2}$ & Albania & 26080 \\
$\mathbf{3}$ & Barrancas & 33507 \\
$\mathbf{4}$ & Dibulla & 30156 \\
$\mathbf{5}$ & Distracción & 15269 \\
$\mathbf{6}$ & El Molino & 8764 \\
$\mathbf{7}$ & Fonseca & 32926 \\
$\mathbf{8}$ & Hatonuevo & 22735 \\
$\mathbf{9}$ & La Jagua del Pilar & 3246 \\
$\mathbf{1 0}$ & Maicao & 154815 \\
$\mathbf{1 1}$ & Manaure & 94272 \\
$\mathbf{1 2}$ & San Juan del Cesar & 37911 \\
$\mathbf{1 3}$ & Uribia & 160631 \\
$\mathbf{1 4}$ & Urumita & 17159 \\
$\mathbf{1 5}$ & Villanueva & 27946 \\
& Total La Guajira & 902386 \\
\hline
\end{tabular}

Fuente: Plan de desarrollo departamental 2012 - 2015 "La Guajira Primero"

Tabla 4

Población a encuestar

\begin{tabular}{ccc}
\hline Ítem & Municipios & Población 2013 \\
\hline $\mathbf{1}$ & Riohacha & 31 \\
$\mathbf{2}$ & Albania & 3 \\
$\mathbf{3}$ & Barrancas & 4 \\
$\mathbf{4}$ & Dibulla & 4 \\
$\mathbf{5}$ & Distracción & 2 \\
$\mathbf{6}$ & El Molino & 1 \\
$\mathbf{7}$ & Fonseca & 4 \\
$\mathbf{8}$ & Hatonuevo & 3 \\
$\mathbf{9}$ & La Jagua del Pilar & 0 \\
$\mathbf{1 0}$ & Maicao & 20 \\
$\mathbf{1 1}$ & Manaure & 12 \\
$\mathbf{1 2}$ & San Juan del Cesar & 5 \\
$\mathbf{1 3}$ & Uribia & 21 \\
$\mathbf{1 4}$ & Urumita & 2 \\
$\mathbf{1 5}$ & Villanueva & 4 \\
& Total a encuestar & 118 \\
\hline
\end{tabular}

Fuente: Los Autores

orientado al ámbito de las Ciencias Sociales denominado SPSS por sus siglas en inglés (Statical Package for the Social Sciences), se procedió al cálculo respectivo del coeficiente de confiabilidad Alfa de Cronbrach, cuyos resultados fueron los siguientes: cuestionario No. $1=0.94$ y cuestionario No. $2=0,92$. De esta manera, se concluye que los instrumentos tienen una fiabilidad respetable, ya que superan el 0,80 .

\section{Resultados}

Con relación a la identificación de los ingresos que el departamento y los municipios han recibido por concepto de regalías a partir del 2005 (ver Tabla 5), se obtuvo un valor para la media aritmética de 3,80 , el cual está incluido en el intervalo que define al rango II, de la categoría Con alta frecuencia. De este resultado se infiere que las instituciones que reciben regalías por explotación de carbón, gas y sal obtienen ingresos de manera puntual, sin inconvenientes y que estos son empleados en su totalidad en la inversión social, tal como lo exige la normatividad vigente.

Por su parte, en lo referente a la identificación de las políticas públicas relacionadas con el Sistema Nacional de Regalías (ver Tabla 6), esta dimensión obtuvo un valor para la media aritmética de 4,30, el cual está incluido en el intervalo que define al rango I, de la categoría Con muy alta frecuencia, de lo cual se interpreta que las instituciones que reciben regalías por explotación de carbón, gas y sal tienen conocimiento de la norma, la aplican con ética, responsabilidad social y son muy honestos y transparentes a la hora de realizar las inversiones sociales y económicas en beneficio de la comunidad.

También, con relación a la identificación de los efectos del nuevo Sistema General de Regalías en el departamento de La Guajira (verTabla 7), esta dimensión obtuvo un valor para la media aritmética de 3,5 el cual está incluido en el intervalo que define al rango II, de la categoría Con alta frecuencia, de lo cual se interpreta que las instituciones que reciben regalías por explotación de carbón, gas y sal hacen inversión social, realizan seguimiento y control a los contratos, aunque no estuvieron de acuerdo con la reestructuración de la norma.

Al realizar la encuesta a la comunidad sobre los beneficios sociales que han recibido por parte de los entes municipales y departamentales con recursos de regalías, de acuerdo a la normatividad vigente, en relación a mortalidad infantil, salud de la población, educación básica, agua potable y alcantarillado, las respuestas obtenidas reflejan lo siguiente:

a) En el departamento de La Guajira la mortalidad infantil es bastante apremiante, puesto que mientras a nivel nacional esta tasa asciende a un total de 15,92 muertes por cada 1000 nacidos, para el departamento de La Guajira, aunque ha venido en descenso, esta tasa supera el doble a la nacional, es decir 32.67 muertes por cada 1000 nacidos. Lo anterior es corroborado con los datos obtenidos en la encuesta. 
Tabla 5

Dimensión de los ingresos recibidos por regalías

\begin{tabular}{|c|c|c|c|c|c|c|c|c|c|c|}
\hline \multirow{3}{*}{$\begin{array}{c}\text { Alternativas } \\
\text { Siempre }\end{array}$} & \multirow{3}{*}{$\begin{array}{c}\text { Rango } \\
\\
5\end{array}$} & & & \multicolumn{7}{|c|}{ Indicadores } \\
\hline & & \multicolumn{2}{|c|}{$\begin{array}{c}\text { Regalías } \\
\text { del } \\
\text { carbón }\end{array}$} & \multirow{2}{*}{$\begin{array}{c}\text { Promedio } \\
3,2\end{array}$} & \multicolumn{2}{|c|}{$\begin{array}{c}\text { Regalías } \\
\text { del gas }\end{array}$} & \multirow{2}{*}{$\begin{array}{c}\text { Promedio } \\
3,2\end{array}$} & \multicolumn{2}{|c|}{$\begin{array}{l}\text { Regalías } \\
\text { de la sal }\end{array}$} & \multirow{2}{*}{$\begin{array}{c}\text { Promedio } \\
3,2\end{array}$} \\
\hline & & 10 & 63 & & 10 & 63 & & 10 & 63 & \\
\hline Casi siempre & 4 & 0,7 & 5 & 0,2 & 0,3 & 2 & 0,1 & 1 & 1 & 0 \\
\hline Algunas veces & 3 & 0 & 0 & 0,0 & 0 & 0 & 0,0 & 0 & 0 & 0 \\
\hline Casi nunca & 2 & 5 & 31 & 0,6 & 5 & 31 & 0,6 & 1 & 1 & 0 \\
\hline Nunca & 1 & 0,3 & 1 & 0,0 & 0,7 & 4 & 0,0 & 4 & 25 & 0,3 \\
\hline Total & & & & 4,0 & & & 3,9 & & & 3,5 \\
\hline $\begin{array}{l}\text { Promedio } \\
\text { Categoría }\end{array}$ & & \multicolumn{9}{|c|}{$\begin{array}{l}3,80 \\
\text { alta frecuencia }\end{array}$} \\
\hline
\end{tabular}

Fuente: Los Autores

Tabla 6

Dimensión Políticas Públicas

\begin{tabular}{|c|c|c|c|c|c|c|c|c|c|c|}
\hline \multirow[t]{2}{*}{ Alternativas } & \multirow[t]{2}{*}{ Rango } & & & \multicolumn{7}{|c|}{ Indicadores } \\
\hline & & \multicolumn{2}{|c|}{$\begin{array}{l}\text { Conocimiento } \\
\text { de la norma }\end{array}$} & Promedio & \multicolumn{2}{|c|}{$\begin{array}{c}\text { Aplicación } \\
\text { de la } \\
\text { norma }\end{array}$} & \multirow{2}{*}{$\begin{array}{c}\text { Promedio } \\
4,1\end{array}$} & \multicolumn{2}{|c|}{ Transparencia } & Promedio \\
\hline Siempre & 5 & 5 & 32 & 1,6 & 13 & 82 & & 8 & 52 & 2,6 \\
\hline Casi siempre & 4 & 6 & 37 & 1,5 & 2 & 12 & 0,5 & 6 & 36 & 1,4 \\
\hline Algunas veces & 3 & 4 & 24 & 0,7 & 1 & 6 & 0,2 & 2 & 12 & 0,4 \\
\hline Casi nunca & 2 & & & 0,0 & & & 0,0 & & & 0,0 \\
\hline Nunca & 1 & & & 0,0 & & & 00 & & & 0,0 \\
\hline Total & & & & 3,8 & & & 4,8 & & & 4,4 \\
\hline Promedio & & & & & & & 1,3 & & & \\
\hline Categoría & & & & & Con & huy al & ta frecue & & & \\
\hline
\end{tabular}

Fuente: Los Autores

Tabla 7

Dimensión efectos del nuevo sistema general de regalías

\begin{tabular}{|c|c|c|c|c|c|c|c|c|c|}
\hline \multirow[t]{2}{*}{ Alternativas } & \multirow[t]{2}{*}{ Rango } & & & \multicolumn{6}{|c|}{ Indicadores } \\
\hline & & \multicolumn{2}{|c|}{$\begin{array}{l}\text { Reestructuración } \\
\text { del SGR }\end{array}$} & Promedio & $\begin{array}{l}\text { Inversión } \\
\text { social }\end{array}$ & \multirow{2}{*}{$\begin{array}{l}\text { Promedio } \\
3,2\end{array}$} & \multicolumn{2}{|c|}{$\begin{array}{l}\text { Seguimiento } \\
\text { y control }\end{array}$} & Promedic \\
\hline Siempre & 5 & 2 & 12 & 0,6 & 1063 & & 10 & 63 & 3,2 \\
\hline Casi siempre & 4 & & & 0,0 & $6 \quad 37$ & 1,5 & 1 & 6 & 0,2 \\
\hline Algunas veces & 3 & 3 & 17 & 0,5 & & 0,0 & 2 & 12 & 0,4 \\
\hline Casi nunca & 2 & & & 0,0 & & 0,0 & & & 0,0 \\
\hline Nunca & 1 & 11 & 71 & 0,7 & & 0,0 & 3 & 19 & 0,2 \\
\hline Total & & & & 1,8 & & 4,6 & & & 3,9 \\
\hline Promedio & & \multirow{2}{*}{\multicolumn{8}{|c|}{$\begin{array}{c}3,5 \\
\text { Con muy alta frecuencia }\end{array}$}} \\
\hline Categoría & & & & & & & & & \\
\hline
\end{tabular}

Fuente: Los Autores 
b) La salud de la población pobre, a pesar de que el Gobierno tiene una cobertura de aseguramiento de su población al sistema general de seguridad social en salud de $96,12 \%$, continua siendo precaria, puesto que son escasas las jornadas o brigadas de salud y el grado de desnutrición en esta población es severo.

c) La educación básica también es crítica. De acuerdo a las estadísticas reveladas por la ministra de Educación, María Fernanda Campo Saavedra, un $30 \%$ de la población en La Guajira es analfabeta, mientras que en el país esta tasa es del $6.6 \%$, siendo la situación más preocupante en el municipio de Uribia, donde un $67 \%$ de la población es analfabeta. De allí que mediante la Ordenanza n. ${ }^{\circ}$ 198 de 2006, se adoptó el Plan Departamental Decenal de Educación 2006-2016 como política pública de educación para el departamento de La Guajira, siendo de carácter indicativo, con los objetivos de garantizar el acceso de la población en edad escolar a una educación de calidad y su permanencia en el sistema educativo durante todos los niveles de la educación preescolar, básica y media.

d) En el departamento de La Guajira, el caso de la escasez de agua potable también es bastante crítico, pues, de acuerdo a las estadísticas presentadas por el Departamento de Planeación, La Guajira está muy por debajo del promedio nacional y los resultados obtenidos en las encuestas son contundentes pues estas reflejan la realidad, puesto que el departamento carece en su totalidad de este vital elemento.

e) Finalmente, en el caso del alcantarillado, también es bastante insuficiente, pues, de acuerdo a las estadísticas presentadas por el departamento de Planeación, La Guajira está muy por debajo del promedio nacional y los resultados obtenidos en las encuestas son claros, pues estos reflejan la realidad, puesto que el departamento carece en su totalidad de este servicio. En zona rural se encuentran los indicadores más deficientes, en tanto que todos los municipios tienen coberturas por debajo del $10 \%$ : Barrancas $(7,79 \%)$, Dibulla $(0,53 \%)$ y La Jagua del Pilar $(0,00 \%)$, para citar algunos ejemplos.

A pesar de los enormes recursos existentes provenientes de las regalías minerales en el departamento de La Guajira, y específicamente en el municipio de Riohacha, destinados por ley al mejoramiento del saneamiento básico ambiental, en el caso del acueducto y el alcantarillado existe una clara discrepancia en cuanto a la información suministrada por los entes gubernamentales y de control, y la percepción de la comunidad y el registro fotográfico aportado.
Los avances descritos por los organismos gubernamentales en referencia a los servicios básicos de agua potable y alcantarillado en sus estadísticas, no se corresponden con la realidad del departamento de La Guajira, en términos generales.

La percepción de la comunidad sobre la inversión de las regalías en saneamiento básico ambiental, como consta en el documento de la encuesta, es concluyente en cuanto al mal uso de dichas regalías, aunque no exista un denuncio formal sobre este hecho y mucho menos un fallo definitorio.

Es evidente la ineficiencia del uso del recurso económico, pues, aunque durante los últimos años las regalías suman la astronómica cifra de más de un billón de pesos corrientes, aún no funcionan en el área urbana y mucho menos en la rural, el acueducto ni el alcantarillado. Un ejemplo de ello es que la frecuencia de suministro del agua es, en promedio, de no más de tres días a la semana, frecuencia que no alcanza las áreas de uso social, siendo, además, agua de mala calidad, según lo certifican la misma Secretaria Departamental de Salud y su Laboratorio de Aguas; asimismo, el alcantarillado es insuficiente, $y$ las aguas servidas se vierten directamente al mar, sin tratamiento previo.

\section{Consideraciones finales}

Indudablemente, una de las conclusiones a las que llega el estudio es que existe una serie de irregularidades en los gobiernos, que hacen de las regalías una torta de la que todos quieren participar, entre ellas se destacan: faltas en la contratación del personal, manejo indebido del presupuesto local, problemas financieros de las instituciones públicas, construcción y ejecución de los planes de desarrollo municipal y departamental, problemas en la documentación legal de los registros, incumplimiento gubernamental, problemas en la formulación y ejecución de proyectos sociales, entre otros.

En este sentido, se recomienda fortalecer los mecanismos de seguimiento, control y denuncia sobre la inversión y la administración de las regalías mineras en todos los niveles, y se sugiere estudiar las propuestas de involucrar al sector productivo local en la inversión y administración de las regalías, al igual que ampliar los incentivos que permitan la deducción de las regalías que deben pagar las empresas a cambio de inversiones importantes en procesos de desarrollo local. 
Las entidades territoriales no están cumpliendo con los aspectos relacionados con la inversión social, por lo tanto, se recomienda:

- Convocar a la comunidad para que desarrolle actividades de control social y participe en la toma de decisiones.

- Designación de interventores administrativos, financieros y técnicos para los proyectos y contrataciones.

- Publicación, por parte de las alcaldías y gobernaciones, de los soportes de los giros, de los proyectos y de los contratos aprobados que comprometen recursos de regalías y compensaciones. Envío a las organizaciones comunitarias de carácter no gubernamental.

Con el análisis y el estudio realizado a las inversiones por concepto de las regalías generadas por la explotación de los recursos mineros en el municipio de Riohacha, se logró determinar que:

- Los recursos provenientes de las regalías mineras son no renovables, puesto que están relacionados con las reservas de recursos mineros con que cuenta el departamento y las cuales están estimadas para treinta $y$ cinco años.

- Los aportes históricos realizados por concepto de regalías mineras indican que el departamento ha recibido más de mil millones de dólares en los últimos años, los cuales no se notan al momento de analizar los beneficios de estos dineros. Cifras estadísticas del Observatorio del Caribe Colombiano y del DANE señalan que al año 2011 , el $64,3 \%$ de la población guajira (unas 562316 personas) vive por debajo de la línea de pobreza, superando considerablemente la media nacional que registra un promedio de $37,2 \%$; y el 35,87\% (313 690 personas), por debajo de la línea de pobreza extrema.

- El NBI compuesto supera el $65.23 \%$, una miseria en la sociedad del $47,10 \%$, vivienda inadecuada el $46,26 \%$, hacinamiento crítico del $46,60 \%$, inasistencia escolar del $15,97 \%$, desnutrición aguda en menores de cinco años del $2 \%$, desnutrición crónica en niños menores de cinco años del $28 \%$, tasa de mortalidad infantil del $32 \%$. Asimismo, entre las causas más frecuentes de defunción están las enfermedades infesto - intestinales y las infecciones respiratorias que, según los expertos, son generadas por la falta de prevención y control en salud y causadas más notoriamente en la deficiencia de los servicios públicos, principalmente de agua potable, con deficiencias para más del $60 \%$ de la población (indicador superior al promedio nacional del $20 \%$ ). Los indicadores estadísticos de enfermedades asociadas con la mala calidad del agua siguen en aumento, la revisión de las diez primeras causas de morbimortalidad del departamento y del municipio muestran una prevalencia de enfermedades relacionadas no exclusivamente, pero si con una fuerte relación directa entre los componentes de multicausalidad con las condiciones de saneamiento básico.

A pesar de que la Ley estipula que los recursos de regalías se deben invertir en mejorar las condiciones de cobertura de recolección de residuos sólidos, principalmente líquidos, por medio de tuberías y conductos, e incluye además el transporte, tratamiento y disposición final de tales residuos, en la capital del departamento la cobertura de alcantarillado no logra un $54 \%$ de la población, además, se presentan problemas de saturación de redes y obsolescencia de los sistemas en la cobertura de los alcantarillados, y carencia de rellenos sanitarios técnicos y no contaminantes.

La vigilancia realizada por los órganos de Control no refleja los resultados que demuestren la eficiencia del manejo de los recursos, no coinciden con el desarrollo o mejoramiento de la calidad de vida de la población riohachera.

Como lo develan las encuestas realizadas, la cobertura del agua potable no abastece el $70 \%$ de la población, el servicio de agua Potable no es constante, se puede contar con él una vez a la semana y únicamente para quehaceres domesticas puesto que no es apta para el consumo, es decir no hay cobertura del agua potable, los más afectados por la falta de estos servicios indispensables son la población rural y los desplazados que viven en las zonas marginales de las ciudad.

\section{Referencias}

Asamblea Nacional Constituyente. (1991). Constitución Política de Colombia de 1991. Gaceta Constitucional No. 116 de 20 de julio de 1991. Bogotá.

Benavides, J., Carrasquilla, A., Zapata, J., Velasco, A. \& Link, M. (2000). Impacto de las regalías en la inversión de las entidades territoriales. Consultoría para la UPME. Bogotá: Fedesarrollo.

Congreso de Colombia. (1994). Ley 141 de 1994. Autores. Congreso de Colombia. (Julio, 18 de 2011). Acto Legislativo No. 05 de 2011. Autores. 
Congreso de Colombia. (2012). Ley 530 de 2012. Autores.

Gaviria, A., Zapata, J. \& González, A. (2002). Petróleo y región: El caso del Casanare. Bogotá: Cuadernos de Fedesarrollo.

Gobernación de La Guajira. (mayo, 17 de 2012). Plan de desarrollo departamental 2012 - 2015 “La Guajira Primero”. Riohacha: Departamento Administrativo de Planeación.

Hernández, R., Fernández, C. \& Baptista, P. (2005). Metodología de la Investigación. México: Mc Graw Hill.

Meisel, A. (2007). La Guajira y el mito de las regalías redentoras. Cartagena: Banco de la República.

Presidencia de la República de Colombia. (octubre, 12 de 1995). Decreto 1747 de 1995. Autores.
Presidente de la República de Colombia. (diciembre, 26 de 2011). Decreto 4923 de 2011. Autores.

Serrano, A., Martínez, M. \& Acened, G. (2016). Formación empresarial hacia la construcción de estrategias de formalización o sustitución de la minería informal en el departamento de Boyacá, estudio de caso municipio Sogamoso. I+D Revista de Investigaciones, 7(1).

Sistema de Información Minero Colombiano. (2015). Producción Oficial de Minerales en Colombia. Recuperado de http://www.simco.gov.co/simco/Estad\%C3\%ADsticas/ Producci\%C3\%B3n/tabid/121/Default.aspx 